\title{
Integrating Digital Nomads in Corporate Structures: Managerial Contemplations
}

\author{
Nicholas Frick \\ University of Duisburg-Essen, \\ Germany \\ nicholas.frick@uni-due.de
}

\author{
Julian Marx \\ University of Duisburg-Essen, \\ Germany \\ julian.marx@uni-due.de
}

\begin{abstract}
The digitization of the world of work affects individuals and organizations alike. Across industries, technological and structural progress offers new potential for individuals to re-organize their work independently of time and place. In this context, the popularized catchphrase of 'digital nomadism' has become an absorbing blueprint for research on the future of work. However, at this point we do not know how organizations can best react to this emerging shift of employee preferences. In this study, we identify hitherto unknown managerial, organizational, and technological implications of integrating digital nomads into corporate structures. The results of expert interviews with executives from various industries shed light on barriers and motivators for corporations to recruit, lead, and retain digital nomads as part of their workforce. Ultimately, we found managers to wrestle with paradoxical attitudes towards digital nomad integration by clearly advocating the flexibilization of working models but resisting cultural change.
\end{abstract}

\section{Introduction}

In today's economy, technological advancements constantly and almost inevitably reshape how organizations conceive business models, products and services, or marketing measures. However, digitization does not only reform economic activity in terms of business processes, but also spurs alterations in the organization of individual work [12]. As Artificial Intelligence or the Internet of Things automatize many operational procedures, highly skilled knowledge workers increasingly account for the bulk of human resources of many corporations. As knowledge work is almost always independent of time and location, professionals progressively pursue the idea of digital nomadism, that is, combining digital work and lifestyle choices [16]. By means of digital collaboration and communication systems, digital nomads monetize their skill sets independently and practice perpetual traveling and geo arbitrage, i.e. receiving remuneration based on western standards while maintaining living expenses in emerging countries [19]. In order for corporations to keep up with the individual preferences of highly skilled professionals, it becomes indispensable to create new working models that allow the integration of digital nomads.

In a recent study, Kong et al. [7] emphasized that marrying corporate structures with digital nomad work may be doomed to failure due to substantially differing values. At the outset, the digital nomad movement evolved around the experiences of freelancers, contract work, and digital entrepreneurs and corporate work was simply not part of the equation. Consequently, extant work on digital nomadism has a strong focus on freelancing and the relationship between digital nomads and corporates from a principal-client and not an employee-employer perspective. However, the mainstreamization of digital nomadism and hundreds of thousands of professionals practicing digital nomadism [16] has led to an increasing overlap between location-independent work and corporate structures. As recent studies show, however, this liaison rests on shaky ground due to an existing mismatch of expectations, values, and structures [7, 10]. In this context, the COVID-19 pandemic and its implications have amplified the reassessment of working models and underscore the tension between 9 to 5 corporate work and the hypermobile digital knowledge worker [20]. Therefore, research is needed that identifies factors of rapprochement and helps narrowing the divide between the two conceptions of work. Existent work on this matter focuses on the perspective of digital nomads, or at least, develops theory based on sampling experts from the digital nomad camp. In order to paint a complete picture, however, it is imperative to scrutinize the corporate point of view and include management and executives in the debate. 
Based on this argument, this study rests upon the following research question:

RQ1: What are motivators and barriers for corporate actors to deploy digital nomad work?

To answer this question, we conducted semistructured interviews $(\mathrm{N}=10)$ with executives from sectors such as IT, production and service industry, and retail. Our study attempts to make a first foray into the examination of digital nomadism from a corporate standpoint. To this end, we identify motivators and barriers that may help scholars to build more robust theory in the emerging field of mobile digital work and helps practitioners to improve their efforts in recruiting, leading, and retaining digital nomads as an integral part of their workforce.

The study is structured as follows. In a literature review section (section 2), we provide an overview on the status quo on digital nomad research and explain the existent work we build our empirical study on. Subsequently, we outline our qualitative research design (section 3), present the findings of our expert interviews (section 4), and discuss them alongside important implications (section 5). We conclude in section 6 with contributions, limitations and an outlook for future research.

\section{Literature Review}

\subsection{Digital Nomadism}

This emerging phenomenon in the context of digital work refers to professionals who use the Internet while perpetually travelling [15]. Digital nomads are mostly knowledge workers from Western countries who combine individual lifestyle choices with digital work. To this end, they use mobile information systems to work remotely [18]. This allows digital nomads to monetize their skill sets independently and practice a highly individualized lifestyle and benefit from geo arbitrage, that is, receiving payment from western-based contractors while vagabonding from one travel destination to another in a personalized way.

Whereas digital nomadism is largely a result of building an alternative work-identity [11] and the desire to travel the world in a long-term fashion [19], this roving practice may also be economically motivated [17]. With a rising number of coworking spaces, topic-related virtual communities and conferences, digital nomadism has evolved from a subculture to a mainstream phenomenon, and eventually, to an industry.

On the lookout for an explanation for personally engaging in this movement, Schlagwein [15] found that digital nomads have different interwoven value systems explaining their choice of working and living. This scrutiny led to three orders of worth which are (1) inspirational order of worth; (2) civic order of worth; and (3) market order of worth. This alternative workidentity primarily results in self-employed or freelance work, ensuring a maximum of independence but also less social security [10]. Notwithstanding the individual motives of digital nomads, using their example as a paragon for new forms of digital and mobile work, the value we can derive from studying this phenomenon is immense. Consequently, a call for research on this matter in terms of economic, cultural, and technological implications [13, 19] is clearly articulated in the information systems discipline.

In order to broaden the debate and include a corporate perspective on the mobilization of digital work, it is imperative to understand to what extend digital nomad work and corporate structures have crossed paths until this point and why it remains challenging for corporations to increase the range of highly flexible working models.

\subsection{Digital Nomad-Corporate Work}

Prior to the popularization of the digital nomad movement, Chen and Corritore [4] found a positive relationship between organizational support for nomadic behavior and employee job satisfaction. Moreover, it underscored the potential impact of a nomadic culture cultivated in a business environment.

Nonetheless, we are far from seeing this cultural change in most branches. Within the last decade, an exhaustive acceptance of digital nomad working models in the corporate sector never really went beyond several IT companies granting software developers remote work arrangements [16]. Not acknowledging the apparent change in individual preferences of knowledge workers, exemplified by digital nomadism, may cause earnest issues for organizations. First, the increasing demand for knowledge workers intensifies the 'war for talent' across sectors and not only the IT industry. Consequently, organizations are pressured to adapt their working models toward the demands of highly skilled knowledge workers. Second, a large increment in flexible and digital working models may challenge the organization of work altogether, as physical meetings, office space, or company cars become superfluous.

The primary reason for the faltering realization of digital nomads working for corporations, according to Kong et al. [7], is a matter of conflicting corporate and digital nomad values. A lack of understanding each other's preferences, poor implementation or adherence to their respective institutional logics and 
misalignment between their worldviews so far impede successful association of both worlds. Since we have gained good knowledge about the ideological discrepancies of corporate and digital nomad work, how come the flexibilization of working models in corporate setting remains a problem?

As we know from previous studies, digital nomads seek flexibility and personal independence, but at the same time, constantly try to achieve a sense of stability and establish routines and structure [7]. As for the emerging work identity, which is normally derived from the organizational environment someone is embedded in, Prester et al. [11] made a compelling proposition: the work identity of a digital nomad is shaped by the constant interplay between the forces of (1) gaining professional autonomy and (2) maintaining self-assured stability.

However, the research to date has a large overhang towards the perspective of digital nomads and their preferences. In order to paint a complete picture, it is imperative to add the corporate perspective to the debate. We found Richter and Richter's [13] conceptualization of digital nomad work (figure 1) to be a useful entry point for confronting corporate executives with the digital nomad phenomenon.

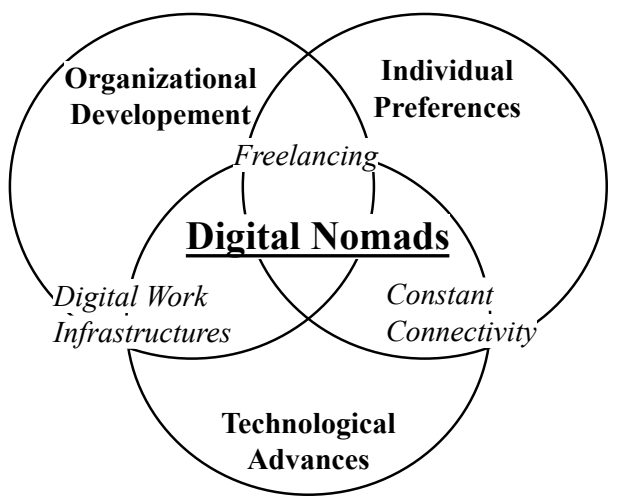

Figure 1. Conceptualization of digital nomad work based on [13].

Whereas previous work emphasized the right circle 'individual preferences', the dimension of 'organizational development' requires additional research. Therefore, our empirical study is based on the conceptual underpinnings of corporate work aiming to specify what determines the junctions with individual preferences and technology.

\section{Research Design}

To determine the status quo of digital nomad work in corporations and to identify motivators and barriers for the integration of digital nomads from a corporate perspective, we build on the threefold concept of digital nomad work [13] to guide our data collection.
We conducted semi-structured expert interviews [9] with executives from multiple German organizations across industries. The audio of the interviews was recorded, paraphrased [14] and analyzed using an abductive coding approach [1].

\subsection{Expert Interviews and Data Collection}

Expert interviews have proven to be an effective research approach to obtain novel knowledge from qualified participants. Especially in less explored domains and within the exploratory phase of research, this method serves as a condensed way for collecting relevant information [3]. The term expert describes an individual who has an advanced knowledge in the investigated field of research [8]. Initially, eligibility criteria were first defined to identify suitable participants. Here, we were looking for individuals working in the management level. Since digital nomadism is an emerging topic, we intended to find a balanced sample involving both management expertise and experts that are familiar with digital work models. Interviewing employees working in key positions within organizations provide "opportunities for expanding the researcher's access to the field" [3]. Moreover, experts should be responsible for employees and have already made hiring decisions.

\begin{tabular}{|c|c|c|c|c|}
\hline $\begin{array}{l}\text { Gen } \\
\text { der }\end{array}$ & Age & Position & Industry & $\begin{array}{l}\text { Dura } \\
\text { tion } \\
\text { (min) }\end{array}$ \\
\hline $\mathrm{m}$ & 29 & $\begin{array}{l}\text { Head of Department } \\
\text { (Emerging Markets) }\end{array}$ & $\begin{array}{l}\text { Energy } \\
\text { Economy }\end{array}$ & $53: 22$ \\
\hline $\mathrm{m}$ & 39 & $\begin{array}{l}\text { Head of Department } \\
\text { (Strategy and Concepts in } \\
\text { Human Resources) }\end{array}$ & $\begin{array}{l}\text { Agriculture } \\
\text { Industry }\end{array}$ & $49: 57$ \\
\hline $\mathrm{f}$ & 27 & Adoption Lead & $\begin{array}{l}\text { Agriculture } \\
\text { Industry }\end{array}$ & $38: 56$ \\
\hline $\mathrm{m}$ & 29 & Business Manager (Sales) & $\begin{array}{l}\text { IT Service } \\
\text { Provider }\end{array}$ & $39: 02$ \\
\hline $\mathrm{m}$ & 32 & $\begin{array}{l}\text { Business Manager } \\
\text { (Machine Learning } \\
\text { Development) }\end{array}$ & $\begin{array}{l}\text { IT Service } \\
\text { Provider }\end{array}$ & $37: 21$ \\
\hline $\mathrm{m}$ & 36 & $\begin{array}{l}\text { Business Manager (SAP } \\
\text { Consulting) }\end{array}$ & $\begin{array}{l}\text { Metal } \\
\text { Industry }\end{array}$ & $46: 56$ \\
\hline $\mathrm{m}$ & 34 & $\begin{array}{l}\text { Category Manager (Global } \\
\text { Sales) }\end{array}$ & $\begin{array}{l}\text { Energy } \\
\text { Economy }\end{array}$ & $39: 14$ \\
\hline $\mathrm{m}$ & 36 & Managing Director & $\begin{array}{l}\text { Service } \\
\text { Industry }\end{array}$ & $38: 08$ \\
\hline $\mathrm{f}$ & 31 & $\begin{array}{l}\text { District Manager (Field } \\
\text { Service) }\end{array}$ & Food Retail & $36: 26$ \\
\hline $\mathrm{m}$ & 29 & Division Manager & $\begin{array}{l}\text { IT } \\
\text { Consulting }\end{array}$ & $30: 19$ \\
\hline
\end{tabular}

Table 1. Sample overview of expert interviews. 
This ensures knowledge about how corporations determine selection criteria for potential employees and what type of employee qualities are being valued. Finally, to gain a holistic picture, the sample should cover organizations from a variety of industries as digital nomadism cannot explicitly be assigned to one specific branch. An overview of our sample is outlined in Table 1.

In total, we conducted ten expert interviews with participants across seven industries. We acquired three business managers, two heads of department, one adoption lead, one category manager, one managing director, one district manager and one division manager. Participants were 32.2 years old on average, with two female and eight male experts.

We used an open interview technique [8] as the most applicable way for retrieving valuable data and providing sufficient room for experts to elaborate on their subjective opinions. The semi-structured expert interviews were supported by a prefixed guideline with central questions considering literature from the method of expert interviews and the guiding concept of digital nomad work [13]. We developed questions for each dimension of the concept and structured the guideline accordingly.

In the first interview phase, the interviewer described the interview process to the interviewee. This included an explanation of the interviewee's rights and verbal consent for the interview to be recorded. The researcher then proceeded with the official part of the interview, which included general questions on the expert's characteristics as well as job and company description. This aimed to be an ice breaker to get the participant comfortable with the interview situation and as well to help the interviewer understand the position of the interviewee is working at his or her company. The second phase served as introduction to the topic of digital nomads and nomadism and contained questions about the current knowledge, for instance, what participants associate with the term 'digital nomads' and if they could think of any examples of digital nomadism. This phase ended with a definition on digital nomads to achieve the same level of knowledge among all participants for the remaining parts of the interview. The third phase of the interviews aimed to get an understanding about the status quo of digital work processes within their organizations. Hence, we asked whether the company provides flexible working hours and the possibility of working from home or from location-independent places. The fourth phased relates to the organizational development, i.e. if the company tries to further digitize working models and what influence technological infrastructure, corporate culture, and management style have. Furthermore, we asked for advantages of flexible working models, the risks associated with this and the impact of the COVID-19 pandemic on the digitization of work. In the fifth phase, we asked the participants about their personal preferences, for example, if they wish for more flexibility in their position and associated risks of new working models. The last phase contained specific questions about digital nomads and nomadism, e.g. whether digital nomadism is just a trend, what kind of skill set is required for digital nomads and how they could alter the culture in organizations. As the last two questions of this phase, we asked the experts whether they think that companies need to hire digital nomads and who they would hire if they could choose between a regular employee and a digital nomad. The interview concluded with the possibility for the interviewee to ask further inquiries followed by a debriefing of the interviewer.

The data was collected between the 8th of April 2020 and the 18th of May 2020 by two researchers. As this period coincides with the COVID-19 pandemic, all interviews were carried out via video call using Google Meet. Since we were interested in the statements of the experts and not in their physical gestures or facial expressions, we deliberately recorded the audio and not the video signal and, respecting data privacy protection, deleted the recordings once the evaluation was finished.

\subsection{Coding Approach and Data Analysis}

As first step of the evaluation, we followed the recommendations in the qualitative assessment of content analysis [14]. Paraphrasing the data reduced the volume by removing unnecessary words to form short and concise sentences. We therefore listened carefully to the interview recordings and paraphrased the content of the experts' statements. Afterwards, we generalized and reduced the content to comprehend and interpret the meaning of the explanations. The analysis of the data was performed using thematic analysis by paraphrasing all interviews shortly after they were conducted.

The data was coded using MAXQDA (version 18) following a deductive-inductive procedure. Deductive categories were derived from theory and new categories were formed inductively [6]. This can be considered as an abductive research design as we started with a conceptual framework [13] and analyzed our data in tandem to the framework [1]. Within the categorization process, we classified the interview data into the three main dimension of digital nomad work, organizational development, individual preferences and technological advances, with the major aim of identifying the status quo as well as motivators and barriers for applying digital nomads in 
organizations. This research approach can be classified as a descriptive procedure since experts are describing the current situation, e.g. what has happened or what is happening now [2]. Considering prior research [5], we used open coding, and thus, paragraphs or sentences as coding units. We coded the material either in-vivo or with simple phrases (2nd order themes) to describe the specific section and further classified them under the dimensions of status quo, motivators or barriers (1st order themes) which we have identified from the interview data itself. For distributing the effort, gaining different perspectives on the qualitative data and ensuring intercoder reliability, the coding process was conducted collaboratively by two researchers. Since the interviews were conducted with German participants, excerpts have been translated into English for the reader's convenience.

\section{Results}

The results are structured along three dimensions. Organizational development explains that, on the one hand, companies may create value and strategic advantage of employing digital nomads but, on the other hand, the execution remains an ambiguous approach. Individual preferences describe the qualities of flexibility and mobility that are important for digital nomads. However, this pliable lifestyle demands finding a suitable balance between stability and autonomy. Finally, technological advances characterize not only the possible increasement of productivity due to collaborative systems but the reliance on certain technology as mobile devices or broadband. In the following, we present our findings according to the status quo as well as potential motivators and barriers for digital nomads in organizations along the three dimensions.

\subsection{Status Quo}

The status quo describes the current situation of the digital and flexible working environments in organizations.

As part of the organizational development, companies have implemented flexible working hours for their employees and further generally provide the opportunity of working from home if the technical foundation is available. However, enterprises are designed for personal contact and employees partly do not desire to work from home but rather prefer a permanent office space. Organizations tend to remain with classic employment arrangements. Experts explain this due to the generation gap and oldfashioned hierarchical structures. Although external service providers, for instance consultants and freelancers, are deployed for various tasks in organizations, digital nomads are not to be found among them. However, companies are generally open to digital nomadism, but also claim that employees should initiate such new work models themselves and deal with associated conditions: "Whoever wants to do this has to take care of it himself. What do I need to do to make it happen? [...] What can I do to acquire a project in another city? Is this person prepared to accept any sacrifices?" (E10).

Flexible and digital work is already established within companies, the demand for further flexibility as individual preference for employees is not necessarily existent. Experts describe that this condition is strongly related to the specific manager and leadership style: "As a manager, I ask myself, how do I lead under classical conditions and how do I lead in virtual environments? Today, my style is described by a lot of trust and openness as well as delegating responsibilities. But then employees must also be willing to take over these responsibilities" (E4). Furthermore, there is also sufficient flexibility for the management level and increased freedom in the form of digital nomadism is not requested.

To what extent it is possible to rise the flexibility of employees at the workplace and what the constitutes the concept of digital nomadism is partly unknown. However, experts claim that that there is a recognizable shift towards digital nomad working models. Individuals working as nomads need to possess digital expertise and a high degree of selfdiscipline since communication and collaboration with colleagues exclusively takes place virtually and the boundaries between the work sector and private life become blurry. Moreover, experts demand that digital nomads need to be open-minded, should be curious about new technologies, work-related task and environments as well as are characterized by a proactive and communicative personality.

Within the scope of digitalization, organizations primarily focus on technological advances as processes, associated infrastructure and how to gain market share. It is less directed at individuals respectively on employees and their personal needs. More flexibility of working arises rather by chance: "Certain processes need to be automated in order to make working from home possible, for example, the digital receipt of invoices. This was done without having working from home in mind, but it helps now. Economic aspects are in in the foreground, but they are also more sustainable" (E6). Experts also report that there are minor problems related to the technical infrastructure. VPN accesses are sometimes overloaded or internet connections are not sufficient what hinders conducting video calls. Overall, the 
experts agree that location-independent digital work is basically possible in a corporate setting.

\subsection{Motivators}

This section explains which factors may induce corporations to introduce or increase the employment of digital nomads.

The experts recognize various opportunities for the organizational development based on the concept of digital nomadism and sensing advantages over competitors, especially if contesting enterprises are not yet deeply digitized. Digital nomads help to ensure that certain tasks are completed more effectively. Nomads working in different time zones may not only cause disadvantages but also generate benefits for certain activities such as the distribution of shifts within a customer service: "For example, digital nomads working with a time difference can also be an advantage. A freelancer can work at times when a customer is available, or the development of code continues without any breaks if several people are working on that" (E8).

Experts argue that the generation of new business models ensuring the long-term success of organizations seems achievable and, in times of crises like COVID-19, still pushes forward digitalization: "There is the possibility for new business models. The way we will be working together is a huge change for transformation. This will make the company successful in the long run" (E2). In addition, experts further identified that the new way of working and employing digital nomads positively influences and motivates inflexible individuals. However, experts differ in their opinion if this cultural transformation entails advantages or whether this poses a threat to the working community and collaboration within.

Organizations further recognize that the concept of digital nomadism comes along with potential cost savings. Digital nomads do not need a permanent workplace in the office and do not have to be equipped with, for instance, a desk and a chair. The level of flexibility and mobility reliefs companies from taking care of analogue equipment. Experts also argue that employees are working from the office even if they show symptoms for a disease. This carries the risk of other employees being infected and absent due to illness. However, since digital nomads have no personal contact with other employees, this lowers the possibility of reciprocal infection. This was particularly mentioned due to the COVID-19 pandemic, but according to experts, applicable in general.

Experts acknowledged various advantages regarding individual preferences in working as a digital nomad. In principle, the concept of digital nomadism leads to an increase in the quality of the individual's life. For example, digital nomads do not need to move for a job, no longer have to shuttle to work every day, are not tied to a specific location or working hours and gain the opportunity to combine travelling and working across the globe. Digital nomadism thus enables different lifestyles and the adaptation to different stages of life. Individuals no longer have to adapt to their job, but vice versa, the job adapts to their preferred life model.

Hiring individuals in organizations as digital nomads further implies promoting trust and confidence as well as less control of the employee. "You should allow employees learning from mistakes by having a culture of constructive criticism and open communication. However, this is only achievable with a certain amount of free space and tolerance" (E1). In addition, organizations and employees need to prepare themselves for novel and probably more intensive social interactions which are not established physically. Within the scope of the COVID-19 pandemic, experts experienced that employees sought contact to each other via unfamiliar avenues. It was described, for example, that employees arranged to meet for a virtual coffee break at a certain time every day, that they had an open virtual meeting room where everybody could attend and that digital gaming nights were organized. This type of digital gatherings seems to be likely as a digital nomad.

Individuals aiming at working as a digital nomad need to be open for personal changes where working results are in the center of attention, not the number of hours at the workplace. However, this holds the potential for increased effectiveness in dependence to the type of personality and provides an appealing lifestyle with a high level of flexibility and mobility.

The application of digital nomadism yields in technological advances and assets for organizations. Digital nomads force companies to engage with (new) technologies, which ultimately creates benefits. For example, experts determined that, as a result of the COVID-19 crises, certain technology was introduced that previously would not have been done at all or only very slowly as part of day-to-day business: "We had to introduce a new VPN, for the huge impact, it was somehow unproblematic. Normally there are 4,0005,000 employees working from home. Now we had to send 60,000 people home within 2 weeks. The ITdepartment deployed a totally new, cloud-based tool for this. It didn't work immediately but after a few days it worked really well" (E7).

However, experts further explained that technological foundations are even available for companies that strongly relate to local customers and infrastructure: "You can download a tool and set it up 
on your device with a landline number from cities in Germany. Even those who are working from Australia. We do this because some customers expect someone to be in a specific city. We have rather hidden the fact that the contact person is somewhere else in the world, we don't peddle that. But you don't have to keep it a secret when appointments are made, or the employees are asked" (E8). Experts describe that digital nomads are driving technological progress within organizations and most of the prerequisites for location-independent work are already existent.

\subsection{Barriers}

In the following, obstacles are explicated that prevent enterprises from employing digital nomads as part of their workforce.

In general, the experts claim that there are several reasons why digital nomadism is not viable within the organizational development of their firm. There are problems regarding the management of individuals, fear of decreasing performance of employees who work independently and expected delays in terms of communication. There is further the apprehension of exploitation of the prepaid trust with the result that employees might work less. In addition, experts believe that older employees will not be convinced by digital and flexible working models and have difficulties in coping with the new circumstances.

Most corporations are characterized by traditional hierarchies, established structures and a fixed mindset. The corporate culture is based on physical presence and traditional employees are expected to be present at certain times and places: "The colleagues should be on site, that's what defines our spirit. We want the people to sit in the office and develop a spirit together. We want to create a cool working environment and atmosphere with table soccer, event area and so on" (E4). Experts fear that the introduction of digital nomads will not create a unified understanding of an interdepartmental corporate culture. Experts likewise show fear of an increased fluctuation due to an increasing flexibility which is not necessarily desired by the company. Employees are able to reorient themselves more quickly, as, for example, there is no need to move to another city anymore. Organizations also explain that they often work in an industry that is strongly described by its physical location. There are branches where it is expected that the staff is present and digital nomads would not be an option. Experts also argue that the expertise of virtual team leadership is not evident within management and is further not taught.

Finally, there are legal issues going in hand with digital nomadism. For example, the concept of digital nomadism as working model must be discussed and approved by the work council. This is frequently a major hurdle, foremost in countries like Germany. Experts state, for example, that there are regularly no harmonized rules for working location independent. In the context of digital nomadism, questions arise such as, what happens if an employee as has an accident while working in another country? Apparently, there seem to be various obstacles that need to be tackled before organizations might consider digital nomadism as a working model. Individual preferences and working as a digital nomad differ from the usual way of working as regular employee in organizations. Working time and location independency must be learned and requires discarding familiar habits which may only be possible to a limited extent.

Experts express that work and private life become increasingly intertwined and lead to an information overload if sufficient self-discipline is missing. Employees feel the need to be constantly available, e.g. 24/7, and no longer have a daily routine, i.e. physical and spatial separation from their work: "When you drive to and from work, you have a separation in time and place, a fixed procedure for the day. Daily routine and leisure time play a secondary role. I naturally ask myself the question, why do I still have to get dressed in the morning?" (E6).

There could be a decrease in performance due to a lack of personal and professional exchange which is dependent on the corresponding personality traits. Introverted individuals are perceived as less appropriate for a digital nomad working model. Experts also fear that digital nomads identify less with the company and that the lack of job security might lead to strive for a permanent job for humans who otherwise wish for a more nomadic lifestyle.

There are varying technological advances in organizations. Not all employees within organizations are equally equipped with the technology. For example, there are employees who still work using a fixed PC and are bound to work in the office, others do have a notebook but are not allowed to take it home. Experts also explain that certain hardware might simply not be available at home for example in manufacturing industries that depend on heavy machinery.

There are also difficulties with regard to accessing certain systems. In the context of data security and protection, external access is limited or restricted and not all employees are granted necessary authorizations. In addition, legacy information systems do not offer the possibility for external access or and might be temporary: "For example, my company switches off the email server at $7 \mathrm{pm}$ in the evening to protect employees" (E6). Experts further complain about the lack of strategic orientation for IT 
and lament about hybrid infrastructure including isolated applications: "We have no IT strategy. We have isolated solutions for individual departments. There are different tools for different areas" (E1). In addition, experts note the lack of possible interpretation of body signals, for example in appointments that take place exclusively digitally: "It's hard to feel what's happening in an appointment. But it is even more difficult to get a sense of what happens in an appointment when the participants are mixed, like a virtual appointment in which one group sits together in the office and others attend from home" (E4).

Finally, we recognized a paradoxical outcome at the end of the majority of the interviews. We explicitly asked the experts the question "Do you think companies need to hire digital nomads these times?" followed by "If you have the choice between a digital nomad and a regular employee, who would you hire under the same conditions and why?". Experts initially confirmed that digital nomads should be hired in principle. However, if they had to choose between the two employees, they would always pick the location- and time-bound employee. One expert illustrates in detail: "I think yes, we should allow ourselves to do this and try it out. For certain tasks, you sometimes don't get anyone else as you are used to that from your business" and further "I would still tend to go for regular employees even though I knew it would be the wrong decision" (E2).

\section{Discussion and Implications}

In terms of the organizational development, our results yielded insights on how digital nomad work may enrich corporate culture and improve operating processes. The fact that digital nomad work can open up opportunities in improving existent business models, e.g. through time zone arbitrage, and for the development of new business models, e.g. digital consulting, stresses the significance of the digital nomadism paradigm for corporate strategy. Our data suggests that, while digitization of work is an ongoing process for corporations, the ramifications of it with regard to individual work are mostly neglected. Executives seem to agree that digital nomads may work as an individual incentive for employee recruitment and motivation, or that it may streamline some existing processes [12]. However, dealing with the impact of digital (nomad) work on the structure, culture, and collaboration within the organization is deferred to an allegedly remote future.

Taking into consideration that our data collection happened to take place during a global pandemic (COVID-19), the examination of digital working models could not be more present for corporations. Experts raised important arguments for fostering digital nomad work, e.g. lower risks of infection even in non-pandemic times. Yet, the prevailing corporate culture and practiced values, as emphasized by Kong et al. [7], remain the most dominant barriers of adjustment toward more nomadic working models. What became apparent in our results is that barriers preventing more corporate digital nomad work are, for the most part, not a matter of circumvented mobility [18]. Apart from supervising jobs in the retail or production sector, digital nomadism is possible without restrictions. However, executives largely seem to argue from a scarcity mentality: fear of losing control, missing trust and insufficient cultural integration [7]. Consequently, the argument against digital nomad work is being made based on the fact that digital nomads do not fit the corporate culture. In fact, experts rarely consider the possibility of adjusting corporate culture in a way so that it better suits the individual preferences addressed by digital nomad work [20].

From an existentialist perspective, digital nomad work may be a vehicle to make organization better serve the individual, instead of vice versa. Experts agree that digital nomad work may improve the quality of life, increase professional independence, and more room for individual lifestyle decisions. At the same time, however, executives largely do not place confidence in the personality traits that are supposedly necessary for being a digital nomad. For instance, extroversion was mentioned to be an important quality of digital nomads. As it turns out, this carnegian assumption is not supported by existent research as professional autonomy is developed from an inner grappling with what work means for oneself [11]. Regardless of personal preferences and work identity, integrating digital nomads in corporate structures comes with legal (e.g. work safety) and ethical (e.g. equal opportunities) challenges that need to be addressed while governing digital nomad work (see overview in table 2). 


\begin{tabular}{|c|c|c|}
\hline Dimension & Motivators & Barriers \\
\hline $\begin{array}{c}\text { Organizational } \\
\text { Development }\end{array}$ & $\begin{array}{l}\text { - } \quad \text { Effective turnaround of work-related tasks } \\
\text { - } \quad \text { Creation of new business models } \\
\text { Employee motivation (provision of attractive } \\
\text { working models) } \\
\text { - } \quad \text { Improved collaboration } \\
\text { - } \quad \text { Cost savings (physical equipment, office space) } \\
\text { symptoms in working environment) }\end{array}$ & $\begin{array}{l}\text { - } \quad \text { Fear of losing control (lack of manageability) } \\
\text { - } \quad \text { Adjuse of trust } \\
\text { - } \quad \text { Missing integration in corporate culture } \\
\text { - } \quad \text { compan fluctuation (missing identification with } \\
\text { - } \quad \text { on physical labor (e.g. large machinery) } \\
\text { - } \quad \text { Missing skillset (e.g. virtual leadership) } \\
\end{array}$ \\
\hline $\begin{array}{l}\text { Individual } \\
\text { Preferences }\end{array}$ & 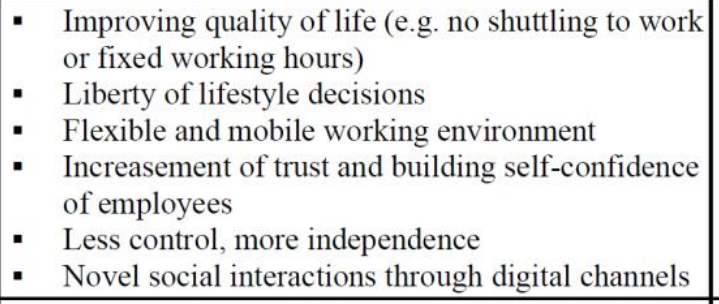 & 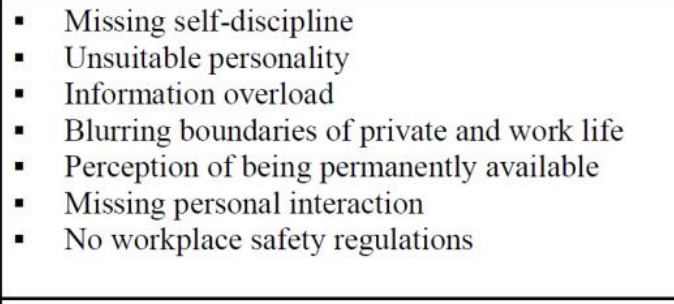 \\
\hline $\begin{array}{c}\text { Technological } \\
\text { Advances }\end{array}$ & $\begin{array}{l}\text { - Organizations are forced to engage with new } \\
\text { technology } \\
\text { - Enforced digitization of communication and } \\
\text { collaboration } \\
\text { - Configurability (e.g. technical concealment of } \\
\text { actual location) }\end{array}$ & $\begin{array}{l}\text { - } \quad \text { No workplac safety regulations } \\
\text { - } \quad \text { Immorying technical equipment among employees } \\
\text { - } \quad \text { Improper or limited access to technological } \\
\text { infrastructure and systems (e.g. legacy IS) } \\
\text { - } \quad \text { Missing IT strategy } \\
\text { Hampered interpretation (e.g. body language) }\end{array}$ \\
\hline
\end{tabular}

Table 2. Motivators and barriers of corporate digital nomad work in three dimensions.

We found technological advances to exert an underlying force that drives the adoption of new working models in organizations. The technological infrastructure, except for IT firms, was only established to combat the challenges coming with COVID-19. However, most experts believe in lasting and accelerated change in terms of work flexibility from the COVID-19 home office measures. Eventually, this will allow the gradual integration of nomad work. Consequently, this requires future work to either rethink the concept of digital nomadism as it - in its current understanding - largely relies on the sovereignty of freelance work rather than being reliant on a corporate entity. In this context, however, digital nomad-corporate work would mitigate one of the biggest obstacles of digital nomads, that is, a financial and social safety net [11].

We found that technology may counteract some of the fears and prejudices towards digital nomad work in corporations. One exemplar for this is the technical concealment of one's location via phone number redirection, which we coined as configurability. Moreover, fostering digital nomad work may promote digitization, a desired outcome of all experts from our sample. At the current state, however, most companies lack the necessary technological infrastructure and IT strategy to enable comprehensive digital nomad work. Digital nomad corporate work, therefore, is at the mercy of corporate values; switched off e-mail servers at night and laptops that are not allowed to leave the office are only two examples of extant barriers.

Conclusively, our study adds the corporate perspective to the phenomenon of digital nomad corporate work. It shows that a successful liaison of digital nomad work and corporate structures requires work on both ends. Therefore, it is imperative for corporations, digital nomads and IS researchers to understand how organizational development, individual preferences, and technological advances can more easily gravitate towards each other.

\section{Conclusion and Future Research}

The present study aimed at the scrutinization of digital nomad work in corporations and possible organizational motivators and barriers in this regard. Whereas existing research has placed a focal point on understanding digital nomad work and its individual inducement, this study offers empirical insights on how this emerging phenomenon is dealt with from an organizational standpoint. Our results suggest that corporations acknowledge the potential value of digital nomad work and entailing benefits for the organization such as employee satisfaction, enforced digitization, or economic gains. Integrating digital nomads, counter-intuitively, does not fail because of physical immobility of organizations. Instead, 
corporations resist cultural change, training in digital work and leadership, and a legal and ethical discussion about digital nomad work.

Our study comes with limitations as our sample may not fully represent all industries and is limited to executives from the middle management level working in German companies. Therefore, some interests within corporations may be over- and some underrepresented in the sample. In addition, not all experts lead teams involving digital nomads, and thus, partly share their attitudes instead of experiences. Moreover, informants may have been biased about the topic of digital work as the interviews took place in amidst the COVID-19 pandemic where digital work was particularly present.

Possibilities for further research are manifold. As our results suggest, additional research may examine the legal and ethical implications of digital nomad corporate work. Moreover, researchers need to broaden the empirical basis around digital nomadism and develop theory to be able to better understand this phenomenon. Eventually, within the IS discipline, digital nomadism will serve as an important exemplar of the digitization of work and may even help to devise what IS research is all about. In fact, digital nomadism revolves around the core of IS research, that is, the interplay of the individual, the organization, and technology.

\section{References}

[1] Alvesson, M., and D. Kärreman, "Constructing mystery: Empirical matters in theory development", Academy of Management Review 32(4), 2007, pp. 1265-1281.

[2] Bear, A., and J. Knobe, "Normality: Part descriptive, part prescriptive", Cognition 167, 2016, pp. 25-37.

[3] Bogner, A., B. Littig, and W. Menz, "Introduction: Expert Interviews - An Introduction to a New Methodological Debate", Interviewing Experts, 2009, pp. 1-13.

[4] Chen, L., and C.L. Corritore, "A Theoretical Model of Nomadic Culture: Assumptions, Values, Artifacts, and the Impact on Employee Job Satisfaction", Communications of the Association for Information Systems 22, 2008.

[5] Gioia, D.A., K.G. Corley, and A.L. Hamilton, "Seeking Qualitative Rigor in Inductive Research", Organizational Research Methods 16(1), 2013, pp. 15-31.

[6] Glaser, B.G., No Preconceptions: The Grounded Theory Dictum, Sociology Press, Mill Valley, 2013.

[7] Kong, D., D. Schlagwein, and D. CecezKecmanovic, "Issues in Digital Nomad-Corporate Work: An Institutional Theory Perspective”,
Proceedings of the 27th European Conference on Information Systems (ECIS), 2019.

[8] Meuser, M., and U. Nagel, Interviewing Experts, Palgrave Macmillan, London, 2009.

[9] Myers, M.D., and M. Newman, "The qualitative interview in IS research: Examining the craft", Information and Organization 17(1), 2007, pp. 2-26.

[10] Nash, C., M.H. Jarrahi, W. Sutherland, and G. Phillips, "Digital Nomads Beyond the Buzzword: Defining Digital Nomadic Work and Use of Digital Technologies", 13th International Conference, iConference, 2018, pp. 207-217.

[11] Prester, J., D. Cecez-Kecmanovic, and D. Schlagwein, "Becoming a digital nomad: Identity emergence in the flow of practice", 40th International Conference on Information Systems, ICIS 2019, (2019).

[12] Richter, A., P. Heinrich, A. Stocker, and G. Schwabe, "Digital Work Design", Business \& Information Systems Engineering 60(3), 2018, pp. 259-264.

[13] Richter, S., and A. Richter, "Digital Nomads", Business \& Information Systems Engineering 62(1), 2020, pp. 77-81.

[14] Schilling, J., "On the Pragmatics of Qualitative Assessment", European Journal of Psychological Assessment 22(1), 2006, pp. 28-37.

[15] Schlagwein, D., "Escaping the Rat Race": Different Orders of Worth in Digital Nomadism, 2017. [16] Schlagwein, D., "The History of Digital Nomadism", International Workshop on the Changing Nature of Work (CNOW), 2018, pp. 1-5.

[17] Schlagwein, D., "Escaping the Rat Race': Justifications in Digital Nomadism", ECIS 2018 Research-in-Progress Papers, 31., 2018.

[18] Schlagwein, D., and M.H. Jarrahi, "The Mobilities of Digital Work: The Case of Digital Nomadism", ECIS 2020 Research-in-Progress Papers, 89, 2020.

[19] Wang, B., D. Schlagwein, D. Cecez-Kecmanovic, and M.C. Cahalane, "Digital Work and High-Tech Wanderers: Three Theoretical Framings and a Research Agenda for Digital Nomadism", Australasian Conference on Information Systems, 2018, pp. 1-12.

[20] Wang, B., D. Schlagwein, D. Cecez-Kecmanovic, and M.C. Cahalane, "Beyond the Factory Paradigm: Digital Nomadism and the Digital Future(s) of Knowledge Work Post-COVID-19 Dialectical Reasoning for Envisioning the Future" Journal of the Association for Information Systems 21(6), 2020, pp. 1379-1401. 